Plasma kinetics issues in an ESA study for a plasma laboratory in space

This article has been downloaded from IOPscience. Please scroll down to see the full text article.

2008 Plasma Phys. Control. Fusion 50074016

(http://iopscience.iop.org/0741-3335/50/7/074016)

View the table of contents for this issue, or go to the journal homepage for more

Download details:

IP Address: 138.100.135.3

The article was downloaded on 27/02/2013 at 12:45

Please note that terms and conditions apply. 


\title{
Plasma kinetics issues in an ESA study for a plasma laboratory in space
}

\author{
B M Annaratone ${ }^{1}$, A Biancalani ${ }^{2}$, D Bruno ${ }^{3}$, M Capitelli ${ }^{3,4}$, \\ F Ceccherini $^{2}$, E Daly ${ }^{5}$, O de Pascale $^{3}$, P Diomede ${ }^{4}$, G D'Ammando ${ }^{4}$, \\ A Hilgers $^{5}$, S Longo ${ }^{3,4}, \mathbf{S ~ M a r c u c c i o ~}^{6}$, J T Mendonca ${ }^{7}$, V Nagnibeda $^{8}$, \\ F Pegoraro ${ }^{2}$ and J R Sanmartin ${ }^{9}$ \\ ${ }^{1}$ Laboratoire de Physique des Interactions Ioniques et Moléculaires, CNRS/Université de \\ Provence, Centre de St Jêrome case 321, 13397, Marseille, France \\ 2 Department of Physics, University of Pisa, via M. Buonarroti 2, 56100 Pisa, Italy \\ ${ }^{3}$ Istituto di Metodologie Inorganiche e dei Plasmi, CNR, via G. Amendola 122/D, 70126 Bari, \\ Italy \\ ${ }^{4}$ Department of Chemistry, University of Bari, via E. Orabona 4, 70126 Bari, Italy \\ ${ }^{5}$ Space Environments and Effects Section, ESA-ESTEC, Keplerlaan 1, 2200 AG Noordwijk, \\ The Netherlands \\ ${ }^{6}$ Department of Aerospace Engineering, University of Pisa, via G. Caruso, 56122 Pisa, Italy \\ ${ }^{7}$ Istituto Superior Tecnico, Av. Rovisco Pais, 1049-001 Lisboa, Portugal \\ ${ }^{8}$ Department of Mathematics and Mechanics, St Petersburg State University, Universitetsky pr. \\ 28, 198504 St Petersburg, Russia \\ ${ }^{9}$ Escuela Tecnica Superior de Ingenieros Aeronauticos, Universidad Politecnica de Madrid, Pza. \\ C. Cisneros 3, 28040 Madrid, Spain
}

Received 26 October 2007, in final form 10 January 2008

Published 4 June 2008

Online at stacks.iop.org/PPCF/50/074016

\begin{abstract}
A study supported by the European Space Agency (ESA), in the context of its General Studies Programme, performed an investigation of the possible use of space for studies in pure and applied plasma physics, in areas not traditionally covered by 'space plasma physics'. A set of experiments have been identified that can potentially provide access to new phenomena and to allow advances in several fields of plasma science. These experiments concern phenomena on a spatial scale $\left(10^{1}-10^{4} \mathrm{~m}\right)$ intermediate between what is achievable on the ground and the usual solar system plasma observations. Detailed feasibility studies have been performed for three experiments: active magnetic experiments, largescale discharges and long tether-plasma interactions. The perspectives opened by these experiments are discussed for magnetic reconnection, instabilities, MHD turbulence, atomic excited states kinetics, weakly ionized plasmas, plasma diagnostics, artificial auroras and atmospheric studies. The discussion is also supported by results of numerical simulations and estimates.
\end{abstract}




\section{Introduction}

A study supported by the European Space Agency (ESA), in the context of its General Studies Programme, performed an investigation of the possible use of space for studies in pure and applied plasma physics, in areas not traditionally covered by 'space plasma physics'. A team of experts, PlaSTET, has been set up to review the possible experiments that could be undertaken and the plasma phenomena they may address [1]. As broad a view as possible has been taken in order to address potential applications in plasma physics, industrial plasma physics and other terrestrial plasma domains including pure plasma physics, as well as astrophysical and solar-terrestrial areas. A preliminary discussion was performed in order to select the most promising experiments and to exclude those issues that can be studied on the International Space Station and therefore do not require a dedicated platform. As a result of this selection work, the following most promising experiments have been further investigated.

- Active magnetic experiment (AcME). The scientific objectives of this experiment are to study magnetic reconnection, instabilities and plasma-body interactions in the electronmagnetohydrodynamic regime and possibly in the pure magnetohydrodynamic regime; also of interest is the dynamics of magnetic bubble expansion. The experiment requires the generation of an artificial magnetic field and a suitable plasma source. Plasma diagnostics should be placed on the ground, on the spacecraft and on a second spacecraft in order to probe the long plasma wake.

- Large discharge facility $(L D F)$. The scientific objectives of this experiment are to study the kinetics of ion-neutral collisions on very large spatial scales and to detect high lying atomic Rydberg states. The experiment requires the release of gas and a RF power antenna. The diagnostics include in situ plasma diagnostics, spectroscopy and a diagnostic package placed on a second spacecraft in order to map distant regions of the discharge.

- Long tether-plasma interactions. In this experiment the tether will be used as an energetic electron source to produce artificial auroral emissions in the E-layer but also for realtime mapping of density profiles for dominant neutral species in the lower thermosphere. More fundamental studies concern the Alfven-wave instabilities and the phenomenon of adiabatic electron trapping. The experiment requires a tether (conductive wire) $10 \mathrm{~km}$ long and plasma diagnostics on the spacecraft.

Two additional experiments that have been discussed in detail during the PlaSTET workshops have not been finally incorporated in the final proposed platform for engineering reasons. The first one is a tether experiment to investigate Alfven front waves, the second one is a proposal for using the relative velocity of two counter-orbiting satellites together with a gas emission to realize a dynamic shock tunnel in earth orbit to simulate realistic conditions for extraterrestrial atmospheric entry. These concepts might be investigated in future studies.

\section{Active magnetic experiment}

Space plasmas provide a natural environment for large-scale experiments in collisionless plasma regimes $[2-4]^{10}$. Active experiments aimed at investigating basic nonlinear phenomena such as magnetic field-line reconnection, magnetic field generation, magnetic vortex dynamics and particle acceleration can be performed under essentially boundary-free conditions. Besides, this is a very interesting new environment in which to test wave scattering plasma diagnostic techniques.

${ }^{10}$ Shabansky V P was quoted in Biriukov et al [4] as the first to propose a satellite with an artificial magnetosphere. 
Within the ESA 'Plasma Laboratory in Space' study a space plasma experiment was proposed $[1,5]$ that consists of a magnetized plasma bubble interacting with the ambient (ionospheric) plasma. In addition, artificial plasmas can be produced by a plasma source (inflated bubble concept). Such an experiment can open up the possibility of studying experimentally the physics of two counter-streaming plasmas in an open and boundary-free environment that cannot be realized in the laboratory where boundary conditions strongly constrain the dynamics of the plasma [6-9]. By controlling the physical and geometrical parameters of the plasma tied to an Earth orbiting satellite we can study the spatial structure of the configurations that the two plasmas can attain, the onset of the instabilities that their relative motion can cause and the plasma turbulence that can result from the development of these instabilities. In the case of non-inflated bubbles the miniaturization of the obstacle from a planet magnetosphere to a satellite magnetosphere leads to plasma regimes that are characterized by dimensionless numbers very different from those that characterize the interaction of the solar wind with the Earth magnetosphere. However, although a physically significant scaling of the magnetosphere solar-wind interaction may not be possible, important information about the non-linear dynamics of collisionless plasmas can be obtained by a relatively simple, satellite-based experiment involving a magnetized plasma bubble tied by a dipole magnetic field generated inside the satellite. Plasmas confined by dipole magnetic fields have good stability properties that have been investigated in laboratory experiments [10-12] and that have been considered for long-term proposals of using thermonuclear fusion for space propulsion $[13,14]$. In this scheme the characteristic size of the bubble, defined as the distance at which the strength of the bubble magnetic field equals that of the ambient field, is essentially determined by the strength of the dipole magnetic field generated inside the satellite. As a consequence, since the dipole field decreases with the third power of the distance from the satellite, it is difficult to confine plasma bubbles with a size more than one order of magnitude larger than the size of the satellite. In an alternative scheme $[15,16]$ the plasma is not confined in a static configuration (in the satellite frame), but a plasma flow is generated by a large neutral particle outflow from the satellite. If the emitted atoms become ionized and 'freeze' the magnetic field in their outward flow, it is possible to obtain much bigger magnetic bubbles. The obvious interest of such a scheme is based on the fact that in this case a wider range of values of the dimensionless parameters that characterize the plasma regime can be obtained. In these inflated bubbles, where the decrease of the magnetic field with distance may be much slower than in the case of a dipole field, most of the current must flow inside the plasma bubble itself. If the currents stop closing through the satellite and the magnetic freezing condition is violated, part of the inflated bubble will become separated from the satellite. However, even in the presence of this disconnection process, the inflated bubble scheme would open up a new type of scenario for active experiments where particle injection and magnetic interactions are combined.

For a non-inflated bubble the most interesting plasma phenomena inside and around the magnetic bubble will occur at frequencies larger than or of the order of the ion gyrofrequency, i.e. outside the range of the standard magnetohydrodynamic approximation. The generation of whistler-type waves by the bubble will be of interest together with the generation of electronmagnetohydrodynamic (EMHD) vortices and vortex streets. It will be possible to produce in a controlled way and to investigate EMHD effects, Hall-dominated, magnetic reconnection events and the development of EMHD turbulence [17-19]. The study of Kelvin-Helmholtz type instabilities on the electron inertia scale are presently of great interest in magnetospheric studies [20]. Such instabilities will be naturally excited in the bubble configuration because of the relative velocity between the magnetic bubble plasma and the ambient plasma. In addition, the fact that the relative velocity is bigger than the ion thermal velocity can be 
used to generate drift waves, lower hybrid waves and sound waves by collective Cherenkov emission and to investigate them in inhomogeneous plasma. This is presently a subject of investigation for the study of the non-linear development of collisionless magnetic field-line reconnection [21]. The restriction to EMHD phenomena can be bypassed with the scheme of the inflated bubble. In particular, MHD physics will become accessible. Besides extending the accessible parameters to the MHD regime, the concept of bubble inflation introduces a new type of physics that deserves investigation in itself, while the magnetic structure of the non-inflated bubble is intrinsically more rigid and thus easier to control and model. These new physics problems involve, aside from the efficiency of the neutral particle ionization and of the successive magnetization of the resulting plasma, the investigation under realistic conditions of the stability of the expanding bubble against magnetic tearing, against current disconnection and against the onset of pressure gradient modes (e.g. ballooning modes), etc. Of particular importance is the understanding of the effectiveness of the momentum and angular momentum transfer from the expanding bubble to the satellite (i.e. determining the resulting force and torque on the satellite and their time variability in the presence of magnetic turbulence). In particular, it will make it possible to investigate the collision between the expanding magnetic field and the ambient magnetic field on spatial scales compatible with the MHD plasma description.

Information on the particle density, energy distribution and on the electric and magnetic fields at the satellite and inside the bubble must be available. They can be obtained by standard plasma diagnostic packages. These data will allow us to investigate specific plasma phenomena, say inductive particle acceleration and sheath acceleration (due to charge separation arising from the fact that electrons are magnetized while ions are not), the formation of turbulent structures, current and vorticity layer dynamics, wake phenomena and electron vortex formation. An additional diagnostic system on a daughter satellite would be instrumental for the investigation of the plasma dynamics outside the bubble (e.g. in its wake). An important diagnostic approach that should be implemented for interpreting this active satellite experiment is the detection of the e.m. waves it radiates. This simple procedure will be highly informative. In addition it will provide a direct tool to sense the dynamics of the magnetic bubble remotely without perturbing it and it is free of the experimental difficulties that the presence of a large magnetic field would pose on the instruments placed on the satellite itself.

As in most modern plasma physics research, numerical simulations based on integrating the Vlasov equation will be instrumental in predicting the main physical processes and in interpreting the experimental data of the interaction between the magnetized plasma bubble and the ambient plasma. Multidimensional particle-in-cell codes must be adapted to the difficult requirements of these simulations: high dimensionality, open boundary conditions and multiple time-scales of interest. Unfortunately it will not be possible in the immediate future to develop a wide scope, physically significant programme of simulations that are three-dimensional both in coordinate space and in velocity space. A simplified phase space description will need to be adopted that is two-dimensional in space and three-dimensional in velocity. This numerical restriction is significant because the scaling of the magnetic field with distance in $2 \mathrm{D}$ and in 3D is different. In figure 1 an example of 2D PIC simulation of the non-inflated bubble [5] is shown: the simulation is performed in the $(x, y)$ plane with $L x=6 \pi$ and $L y=2 \pi$ in electron skin depth units. The Debye length is 0.1 . Open boundary conditions are used in the $x$ direction, while periodicity is assumed in the $y$ direction. The external magnetic field, periodic in $y$, is localized in the central region with typical dimension of the order of a few units. The plasma flows along the $x$ direction at $u=0.05$ in electron thermal velocity units. The simulation mesh grids are $N x=128, N y=32$ for both electrons and ions. The ion to electron mass ratio is 1000 . The external magnetic field is generated by two opposite currents 

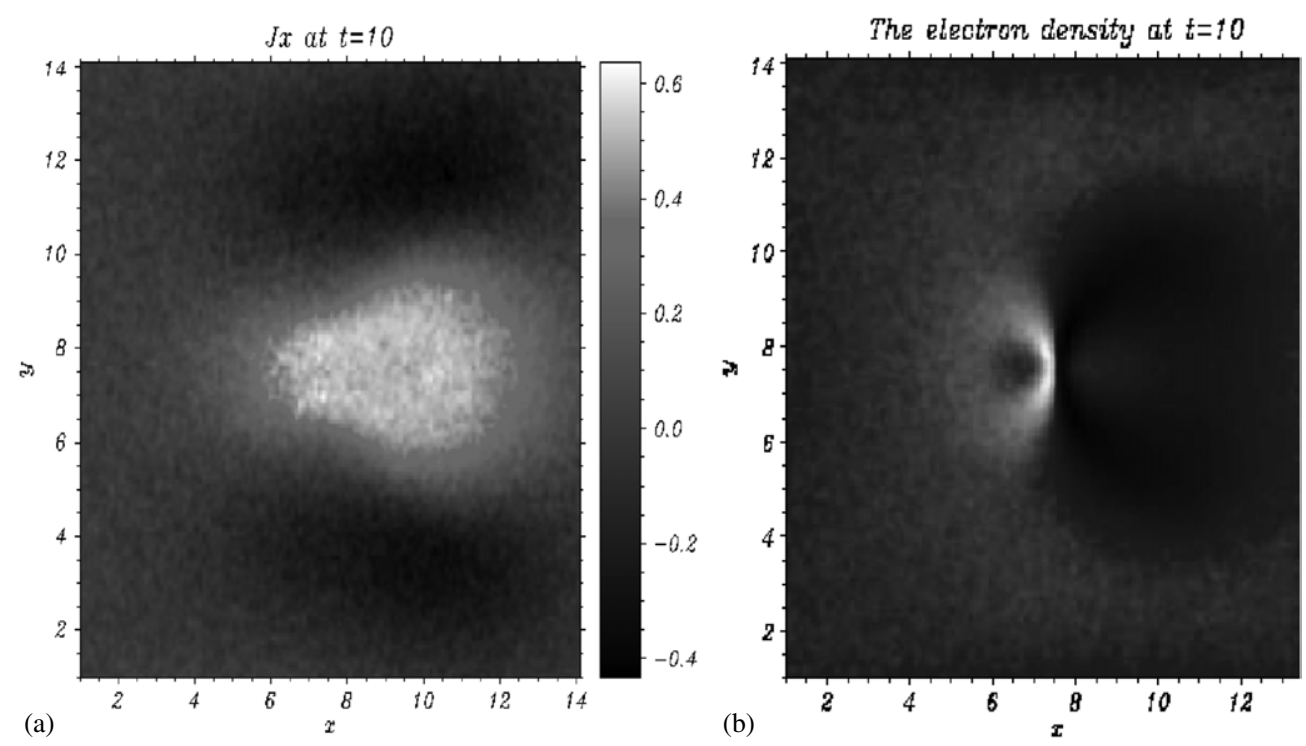

Figure 1. Sample of current density (a) and charge density (b) for 2D PIC modeling for the non-inflated bubble.

along $z$. The equations for the update of particle position and velocity under the effect of locally interpolated electric and magnetic fields are integrated with the Boris method [22].

In figure 2 an example of 3D hybrid simulation of the inflated bubble [16] is shown. The hybrid simulation used in this study is based on the description of the electron component as a fluid, while ions are treated by the PIC approach.

The interest of the more challenging inflated bubble configuration lies not only in the unprecedented large-scale plasma physics (turbulence, instability, wave/plasma) made accessible, but also in the possibility of experimenting with the concept of plasma bubble based sailing to produce thrust on a payload from solar radiation [15, 23, 24].

We have also examined the processes of plasma bubble formation and expansion around a magnetized spacecraft, as proposed for the artificial magnetospheric propulsion scheme. These processes can be described by two coupled equations, one for the neutrals and the other for the ambipolar plasma expansion. Typical length and time-scales were established. Creation of a plasma bubble by photo-ionization of the neutral gas released by the spacecraft is, in principle, an interesting alternative to be considered for this propulsion scheme. This is due to the low degree of ionization of the plasma created by typical plasma sources, such as the helicon sources, installed inside the spacecraft. If possible, this would considerably reduce the weight and the energy needs and simplify the propulsion scheme [25].

The two bubble concepts ask for a very different external environment: a strongly elliptic geostationary transfer orbit (GTO) solution (figure 3) fits the different ambient plasma requirements with a single payload.

\section{Large discharge facility}

The experiment is devoted to the production and study of large-scale (10-100 m) discharge plasmas in open space, including hydrodynamics and atomic physics issues. The peculiarity of these experiments with respect to already performed active plasma experiments is the role 


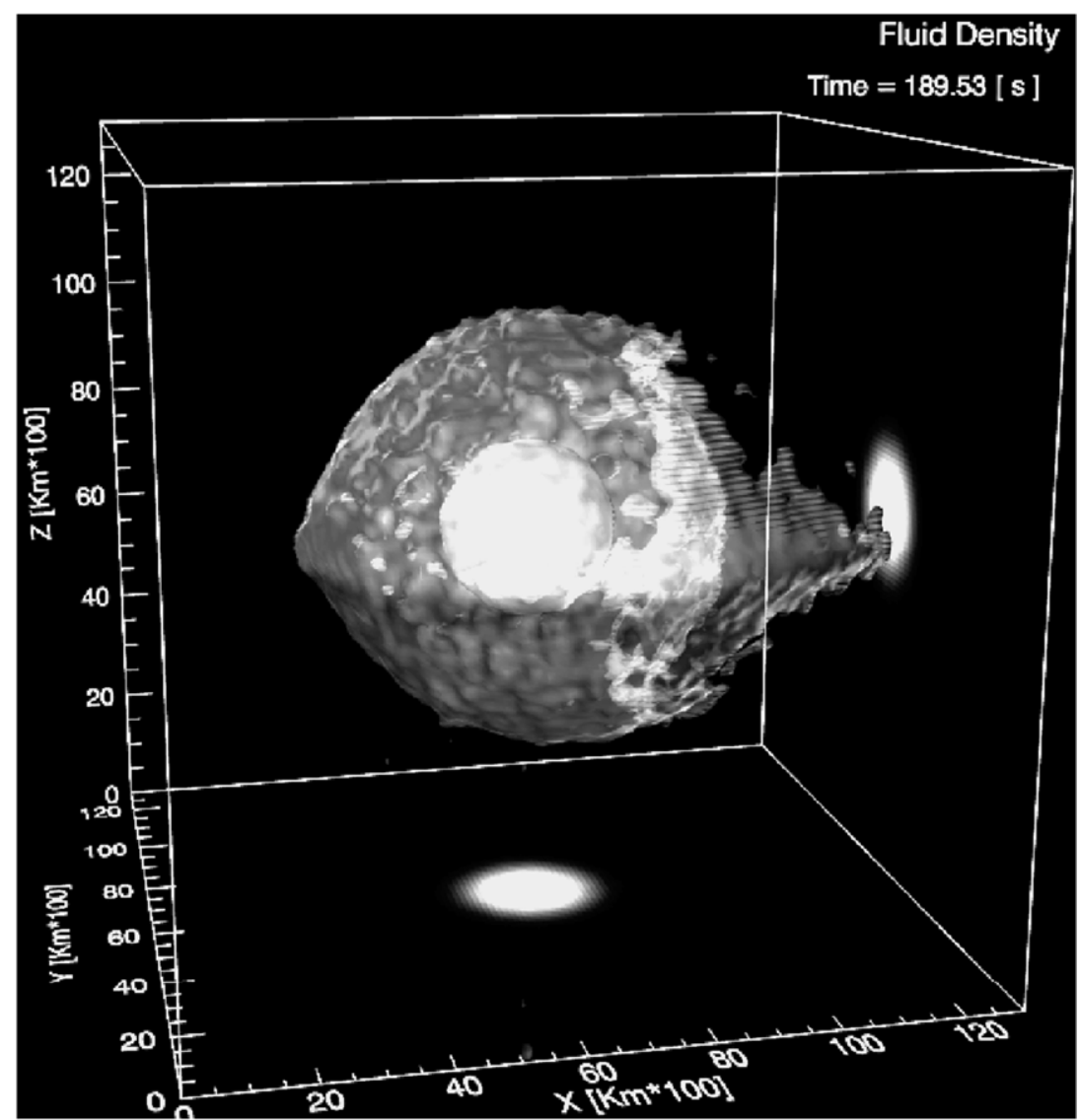

Figure 2. Sample results of 3D hybrid simulation of the inflated plasma bubble.

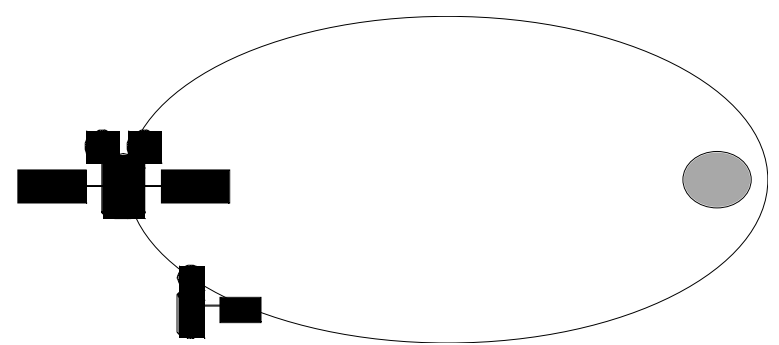

Figure 3. GTO orbit, main payload and detached diagnostic sub-satellite (not in scale).

played by ionization processes and the stress on cold plasma physics, where the collisions of charged species with neutrals are most relevant. Because of this last circumstance, atomic physics and hydrodynamics strongly affect the system, while the variety of collective plasma phenomena is drastically reduced.

Typical laboratory low temperature plasmas are characterized by $p=10-100$ mTorr, $T=300 \mathrm{~K}, L$ (discharge gap) $=$ a few centimeters, $n \sim 10^{15} \mathrm{~cm}^{-3}$, low ionization degree $\alpha \sim 10^{-7}$. 
The analogous space plasma is set up based on a choice of scaling: for example, one can keep approximately the same Knudsen number and $\alpha$. As a result, one gets the following specifics: $L=100 \mathrm{~m}, n=10^{11}-10^{13} \mathrm{~cm}^{-3}$, matter quantity $\sim$ a few moles. The expansion timescale: $\sim 0.1 \mathrm{~s}$ for $\mathrm{He}$ at $100 \mathrm{~K}$. Mass requirement is evaluated elementarily based on the scaling of sound speed and $n$, and at fixed $n \sim M^{1 / 2}$ ( $M=$ molar mass). All requirements can be drastically reduced for a strongly asymmetric emission, jet-like; nevertheless in the following we will stick to the spherical geometry.

Skin depths issues are the essential factor to account for plasma heating; they can be studied by a simple absorption model, assuming collisional plasma heating and atomic gas (He). The local power deposition density can be estimated as [26]

$$
P_{\mathrm{abs}}(r)=\frac{1}{2} \alpha n_{\mathrm{g}}(r) \frac{e^{2}}{m_{\mathrm{e}} v(r)} \frac{1}{1+(\omega / v(r))^{2}} E_{0}^{2}(r),
$$

where $E_{0}$ is the angle-averaged local field amplitude, $\omega$ is the field angular frequency, $v$ is the collision frequency; this last can be estimated as $n_{\mathrm{g}}(r) \sigma \sqrt{8 k T_{\mathrm{e}} / \pi m_{\mathrm{e}}}$, where $\sigma$ is the momentum transfer e-He cross section. The gas density can be calculated assuming that the gas performs an isotropic expansion from a spherical surface of radius $r_{0}$ and that the gas kinetic terminal speed [27] is attained soon:

$$
n_{\mathrm{g}}(r)=\frac{\text { m.f.r. }}{4 \pi r^{2} \sqrt{5 k T_{0} m_{\mathrm{He}}}},
$$

where m.f.r. is the mass flow rate and $T_{0}$ the initial gas temperature. A dipole e.m. field is assumed to relate the angle averaged relative e.m. power $W(r)$ to $E_{0}$. In figure 4 results are shown for m.f.r. $=1 \mathrm{~g} \mathrm{~s}^{-1}, r_{0}=1 \mathrm{~m}, k T_{\mathrm{e}}=1 \mathrm{eV}, T_{0}=100 \mathrm{~K}, \omega=10^{4} \mathrm{~s}^{-1}$ : it appears that most heating occurs in the plasma center, while better results are obtained at lower ionization degrees. In the same picture the effect of changing the m.f.r. in a single case is also displayed.

Since the power absorption law is linear it cannot fix the total heating power, which can be evaluated based on the necessity to sustain $T_{\mathrm{e}}$. A rough estimate of the minimal power required per unit volume based on elastic losses only is $(2 m / M) v n_{\mathrm{e}} k T_{\mathrm{e}}$, which is in the order of a few $\mathrm{mW} \mathrm{m}^{-3}$ (due to the very low gas density and $\alpha$ ) but the real value is much higher and strongly dependent on the additional ionization desired: of course, realistic calculations must include the effect of energy deposition and hydrodynamics on the electron temperature in different positions and the effect of non-Maxwellian electron energy distribution function; methods for such calculations have been reviewed in [28] but calculations proper for this test case are extremely difficult and they have never been attempted.

The necessity of a low ionization degree increases the sensitivity of the experiment to ionospheric plasmas, and as a result the experiment cannot be performed in LEO, but it can be run on most of the envisaged GTO orbit (figure 3).

Preionization issues are critical, but they can be solved using a plasma source.

In terms of diagnostics the best choice is probably the most conservative, based on the experience of earthbound discharge science, i.e. optical emission spectroscopy, Langmuir probes, mass spectroscopy (the daughter payload of figure 3 can provide great help). The diagnostic technique described in section 4 could also be applied in this context.

The large-scale discharge plasma is an ideal environment to study the kinetics of Rydberg states of atoms and molecules very close to the continuum. This is explained based on the Fermi cut-off condition for the principal quantum number $N$ in atomic hydrogen-like states:

$$
a_{0} N_{\max }^{2} \approx n_{\mathrm{g}}^{-1 / 3},
$$

where $a_{0}$ is the Bohr radius. The condition above expresses the contact of valence shells of atoms in the gas. In the large-scale plasma $n$ is very low, lower than any possible achievement 


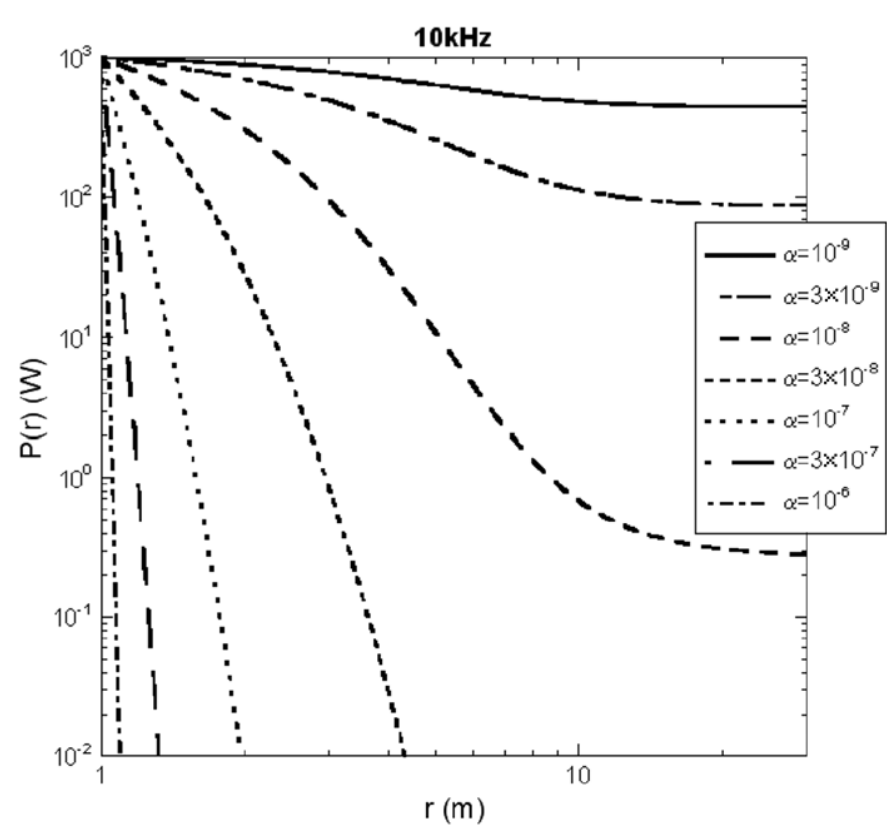

Figure 4. Results from the radial absorption model. a: $\alpha=10^{-6}$, b: $\alpha=10^{-7}$, c: $\alpha=10^{-8}$, $\mathrm{d}: \alpha=10^{-9}$; dashed line: $\alpha=10^{-8}$ with a m.f.r. of $0.2 \mathrm{~g} \mathrm{~s}^{-1}$.

in earth bound vacuum chambers. At the same time, the large plasma extension allows for a significant detectable Rydberg state population along a line of sight. As regards detection, one possibility is the analysis of e.m. emission in the relevant region of radio waves, this emission being produced by spontaneous radiative decay and maybe recombination by a simple dipole antenna. The Rydberg formula gives the recombination line frequency due to transition from high $i$-level to $(i-\Delta i)$ level $(i \gg 1$ and $i \gg \Delta i)$. For $\mathrm{H}$ atom (for such levels other atoms are as $\mathrm{H}$-like atoms and this formula is still appropriate) and $\alpha$-transition from $i$ to $(i-1)$ [29]

$$
v_{i \alpha}=3288052(2 i+1) /\left(i^{2}(i+1)^{2}\right) \mathrm{GHz} \text {. }
$$

Thus, for example, for $i=1000, v=6.58 \mathrm{MHz}$. Ground-based radio-telescopes can only operate at frequencies higher than $10-30 \mathrm{MHz}$ because of ionospheric cut-off. Therefore observations in such a low frequency range is possible only with space radio-telescopes on board spacecrafts which fly at heights above ionosphere $>1000 \mathrm{~km}$.

It is necessary to estimate the intensity of these spectral lines. Some of the problems to be considered to reach this goal are only in minimal part covered by the literature [28, 30-33].

- Calculation of the absorption coefficient in lines especially taking into account induced emission.

- Mechanism of population of high levels (radiative recombination and collisions) and balance equations. Analysis of this problem showed that such a mechanism produces inversed population on high levels: a maser-effect is therefore possible.

- Non-LTE analysis.

- Calculation of the optical thickness of the source of emission (integral along the line of sight).

- Broadening of the lines due to the Doppler effect (thermal velocity and turbulence) and smoothing of the spectrum (lines are very close to each other). 
The study of Rydberg states is of paramount importance for the understanding of collisional plasma physics, in particular energy budget, e.m. emission and transport properties [34] (because of the very high electron impact cross section of highly excited states).

Another issue worthy of consideration is the possibility of using the LDF as a laser source, operating on high gain infrared vibrational transitions ( $\mathrm{CO} 2, \mathrm{CO}, \mathrm{HCN}$ [35]). Of course this proposal needs detailed calculation to check its feasibility. Gain calculations including nonequilibrium electron energy distribution, vibrational kinetics and gas flow, the interrelation of electrical pumping and gas dynamic inversion, have never been done for plasma extended on several meters and such unusual conditions and represent a deserving challenge for discharge plasma theorists.

A comparison can be attempted between the proposed experiment and recent plasma space experiments, in particular CRRES and AMPTE [36], involving collisional and transport features in a weakly ionized medium: in particular, in the former experiments the impurities traced were mostly low IE (ionization energy) metal atoms like barium and lithium. The present experiment focuses on the properties of the artificial plasma as a whole and is not limited to impurity transport in the ambient plasma. In fact, impurity transport here is not the central issue, while we are considering the global aspects of the plasma cloud, including ionization kinetics and rarefied hydrodynamics. Besides, the special attention posed here on the problem of non-equilibrium collisional kinetics, including excited species, is expected to improve our knowledge of weakly ionized plasmas artificially produced in open space. In turn, this knowledge can provide a way to future space technology where large-scale plasmas could play a role in open space, like nanotube production, 'solar sails' surface refining and similar ambitious large-scale surface processing connected to thermal and radiation protection. Furthermore, negative ion production in open space paves the way for energetic atom beam and their application to neutral particle/sail propulsion.

\section{Space resolved measurement of plasma density}

Although much information can be obtained from standard plasma diagnostic packages, nonintrusive means of measuring the plasma density constitute a desirable alternative to the Langmuir probes.

Assume as a scenario a plasma expansion in vacuum. This is relevant for the bubble inflation experiment, section 2, and to the large discharge experiment, section 3, but also for ground applications that range, with different length scales, from laser ablation of materials, to inertial fusion, to plasma after glows and others. The underlying theories are summarized in [37-39]. Charge separation effects in collisionless plasma cannot be properly studied in the laboratory because the asymptotic phase of the expansion develops for $\omega_{\mathrm{pi}} t \sim 100$ [38] that, with velocity of the order of $10 c_{\mathrm{s}}$, gives lengths of 10 to $100 \mathrm{~m}$. Following a more recent publication [39], the relevant parameter is $\bar{T}_{0}=Z k_{\mathrm{B}} T_{0} / \varepsilon_{\mathrm{CE}}$, also equal to $3 \lambda_{\mathrm{D}}^{2} / R_{0}^{2}$, where $\lambda_{\mathrm{D}}$ is the Debye length for the electrons and $\varepsilon_{\mathrm{CE}}$ is the maximum ion energy obtainable from a Coulomb explosion (CE) of a sphere of ions with radius $R_{0}$. With initial plasma density varying from $10^{13}$ to $10^{18} \mathrm{~m}^{-3}$ and a reasonable size source, the parameter $\bar{T}$ scans from 0.1 , almost CE regime, to the hydrodynamic limit. Given the large length available, $\sim 100 \mathrm{~m}$, a range of hybrid regimes are so accessible. Recombination effects and the role of turbulence can also be studied.

Separate spatial and temporal resolved diagnostics will monitor the plasma species, electrons and ions; a mixture of heavy and light ions will add, in a later phase, further interest to these experiments. We could also consider the release of electronegative discharges or 
nanoparticles as produced in methane-hydrogen plasmas. These latter species are negatively charged slow components.

The ion front will be detected by conventional spectroscopy. The analysis of the signal will have to be interpreted in accordance with the reduced density of the exciting electrons.

For the fast electron front the diagnostic is based on the cut-off of electrostatic, longitudinal waves at $\Omega_{\mathrm{pe}}$ and on the plasma-sheath resonance. The latter is one in which the plasma resonates with its adjacent sheath forming a kind of boundary layer to the plasma [40]. At radiofrequencies the sheath behaves essentially as a capacitor, since the smaller number of electrons there has a limited effect. The region between the two electrodes can be described in terms of either (a) an equivalent circuit containing an inductance, together with a vacuum capacitance, or (b) a negative capacitance which is frequency dependent. In the radiofrequency range only the free electrons of the plasma contribute, the negative charge of the particle or of the negative ions does not participate in the process. When different types of plasma and complex plasma co-exist, the equivalent inductance is the series inductance along the path of the radiofrequency current. At certain frequencies the plasma-sheath resonance takes place. For a plain geometry a simplified equation is

$$
f_{\mathrm{R}}=\frac{f_{\mathrm{ep}}}{\sqrt{1+\frac{p}{2 s}}},
$$

where $p$ is the length of the current path in plasma and $s$ is the sheath thickness. In non-uniform plasmas the plasma-sheath resonance gives the plasma density in front of the electrodes, one RF excited with scanning frequency, and the other, return electrode, grounded or driven in the opposite phase. Both the electrodes are placed on the satellite. The cut-off frequency gives the maximum density of the plasma. For frequencies above $\Omega_{\mathrm{pe}}$ the space is capacitive and transmission is possible.

We propose to apply a constant amplitude and variable frequency RF signal to a small electrode on the satellite facing the plasma. Only one electrode is required in this case, the other being the satellite chassis. Two modes of operation are envisaged. In the first the full spectrum is obtained by scanning the RF and recording the current. Alternatively, we can record the dc bias produced. From the identification of the above resonances we can derive the higher density of the plasma (the cut-off frequency gives the maximum density of the plasma) and the ratio $p / 2 s$ is derived in a similar manner as in equation (4.1). In [41] we have used the same geometry as the one proposed in this experiment and measured that the return path in plasma, $p$, is 1.6 times the electrode dimension. This first method of operation is probably too slow with respect to the characteristic time of this experiment.

In a second mode of operation the electrode is kept at a constant frequency and constant amplitude while the density and the sheath thickness vary with time. We can record the current, or the dc bias induced, as a function of time. The chosen fixed frequency will match before the plasma-sheath resonance and after the plasma resonance (corresponding to a later time) from which we can derive the time of a certain plasma density. Repeating the experiment at different frequencies in identical shots we can reconstruct the time evolution of the cloud. Moreover, comparing plasma-sheath resonance in one shot and the plasma resonance in another shot at the same time (or extrapolating from the evolution of these two resonances) we derive in real time the space distribution.

\section{Long tether-plasma interactions}

A conductive tether over $10 \mathrm{~km}$ long, left bare of insulation and electrically floating (i.e. using no electric contact devices at tether ends, current vanishing there) in low earth orbit, would 
be an effective electron-beam source to produce artificial auroras. Because of the large ionto-electron mass ratio, the tether would be biased highly negative by the motional $(\bar{v} \times \bar{B})$ electric field and attract ions over most of its length. Ions impacting with kilo-electron-volt energies would liberate secondary electrons, which would locally accelerate away from the tether, then race down geomagnetic lines and result in auroral emissions in the ionospheric E-layer [42].

Beyond auroral effects proper, observations down the beam from the spacecraft carrying the tether could provide real-time mapping of density profiles for dominant neutral species in the lower thermosphere, of interest for numerical simulations of the atmosphere lying below and in re-entry predictions [43]. Chemical, thermal and transport processes at 120-160 km altitudes, a region too low for in situ satellite exploration and too high for balloons (while rockets fly by in too short times), are a subject of outstanding interest.

Since 1969, electron-beam sources on board spacecraft (S/C) have been used in active experiments for producing artificial auroras but such 'standard' e-beams are marred in several ways. Beam firing affects the S/C potential serving as ground for the beam source. Gross perturbations produced by the intense emission in plasma around the $\mathrm{S} / \mathrm{C}$ affects the beam itself, and the luminous glow arising from electron bombardment in the return current contaminates optical instruments. Beam flux up to two orders of magnitude greater than in the strongest natural auroras compensates for the thinness of the emitting layer and makes ground observation possible; high-flux beams, however, produce hot electrons and plasma fluctuations and are distorted by non-linear plasma interactions.

The tether e-beam is free of S/C charging problems (no current flows at tether ends) and free of plasma-interaction problems, beam density and flux being low. Also, beam emission takes place far from any instrument. The tether low-flux, thin beam, however, exhibits brightness for ground observation as low as 1 Rayleigh, light sources in the night sky masking such signals (though the signal-to-noise ratio might possibly be increased by pulsing the hollow cathode with phase-locked detection). On the other hand, brightness is much greater for observation from the spacecraft, also allowing continuous measurements. This is impracticable for the thin cross section of a standard beam, but the tether beam has a cross dimension of the order of the tether length, of the order of $10^{4} \mathrm{~m}$.

Each point in the tether, which is deployed downwards, emits monoenergetic secondary electrons, but both electron flux and energy increase almost linearly with the distance $h$ from the top of the tether. Observations from the spacecraft along any straight line at angle $\psi$ from the magnetic field direction, covering some altitude range $\Delta z$ over the emission region, would mix $h / z$ effects. The narrow footprint of the beam in the emitting layer will then show a peak in brightness versus angle $\psi$, allowing tomographic analysis of the signal.

As beam electrons move in helical paths down magnetic field lines, they lose energy in inelastic ionization and excitation collisions, followed by prompt photon emission in case of allowed transitions; electrons are also scattered in elastic collisions with air molecules, which modify the pitch-angle distribution and broaden the beam through diffusion across magnetic lines. The beam dwell-time at any particular point will be a few tens of milliseconds, which is much too short for beam-induced ionization to affect the electron density. On the other hand, the population of neutrals in excited states with prompt emissions through allowed transitions (lifetimes $\sim 10^{-7} \mathrm{~s}$ ) do reach a steady state, emission rates then being proportional to excitation and ionization rates.

The narrow emission footprint of the beam, which covers a line-of-sight range $\Delta \psi \approx 6^{\circ}$, shows a peak in brightness that is about $10^{2}$ Rayleigh for prominent bands and lines, well above background noise. Interference filters could be used to register emissions at the $391.4 \mathrm{~nm}$ or the $427.8 \mathrm{~nm}$ spectral bands for $\mathrm{N}_{2}$ and at the $777.4 \mathrm{~nm}$ and $844.6 \mathrm{~nm}$ lines, with definite branching 
ratios, for $\mathrm{O}_{2}$ and $\mathrm{O}$. The narrow footprint of the beam would allow spectral separation of the incoming radiation by a grating, to form non-overlapping images at different wavelengths.

The tether operates at nighttime, with power supply and a hollow cathode at the S/C off, for current to vanish at both tether ends. Orbit altitude should lie around the ionospheric $F 1$-layer maximum; an altitude just over $300 \mathrm{~km}$ is suggested by considerations on both safety of the International Space Station and low air drag. A moderate orbit inclination is favored by the need for a large motional electric field (independently, magnetic dip angles around $45^{\circ}$ make for a broader brightness peak). The geomagnetic field exerts a Lorentz drag on the electron current, which flows downwards throughout the tether in case of an eastward moving S/C. Both power and the hollow cathode at the top would be on at daytime, to partially reverse the current and reboost the S/C once per orbit. This would make the tether an autonomous e-beam source, which might change its own orbit when desired.

The tether would be an aluminum thin tape, both low density-to-conductivity ratio and low area-to-perimeter cross-section ratio keeping tether mass down; in the convenient orbitalmotion-limited (OML) regime, current collected per unit tether length is proportional to the perimeter [44]. A tape of width $w$ and length $L$ will collect ion current scaling as $w L \times \sqrt{L}$; for yield taken roughly proportional to bias, and full bias $\propto L$, the emitted current scales as $w L^{3 / 2} \times L$. With footprint dimensions $L$ and $\sqrt{L}$ at the E-layer, the beam flux, determining brightness, will scale as $w L$, while the line-of-sight emission rates will roughly scale as $w L \times L$, greater energy allowing deeper penetration down the E-layer. Both large tether width and length are thus required. A wide tape would also reduce the probability of cuts by debris, but OML current collection limits $w$ to less than about $25 \mathrm{~mm}$.

Although tape mass can be reduced by making it thinner whatever $w$ and $L$, the total system mass presents a (broad) minimum at a particular ratio $L^{3 / 2} / \delta$. For too thick a tape, 'hardware' mass, which accounts for spacecraft, end-mass/deployer, and tether itself, and is taken as a few times the tether mass $(\sim w L \delta)$, would be dominant ( $\delta=$ tape thickness). At too low $\delta$, however, the power-subsystem mass is dominant: a tape very thin and thus having a large ohmic resistance requires a large solar array to push current through at daytime. Thrust power is proportional to motional field $\times$ current $\times L$, thus scaling as $w L^{5 / 2}$ for OML current $\left(\sim w L^{3 / 2}\right)$. The power-subsystem/hardware mass ratio would then scale as $L^{3 / 2} / \delta$. Ohmic effects on day thrust are gauged by the ratio between OML current $\left(\sim w L^{3 / 2}\right)$ and short circuit current $(\sim w \delta)$, which also scales as $L^{3 / 2} / \delta$.

For $L=15 \mathrm{~km}$ and typical orbit day/night conditions, system-mass minimum occurs at $\delta=0.12 \mathrm{~mm}$. A tape of width $20 \mathrm{~mm}$ would have a mass of about $97 \mathrm{~kg}$; for hardware mass four times as large, total mass would be about $950 \mathrm{~kg}$. Hollow cathode expellant mass need only be considered in the case of missions beyond half a year. An implementation of this experiment is currently planned to be flown on the Japanese S-520-25 rocket experiment to be launched in the summer of 2009.

\section{Conclusions}

This study identified several novel plasma experiments to provide improvements of understanding of plasma physics and chemistry under controlled conditions in a range of parameters not accessible on the ground. They deal with plasma expansion either in magnetized or non-magnetized regimes, streaming plasma interaction with plasma bubbles, and creation of auroras by a tether generated large electron beam. Besides the fundamental plasma physics and chemistry aspects, at least two of them have potential interest for application (i.e. magnetic sailing with a large magnetic bubble and upper atmosphere diagnosis by the tether experiment). Although the experiments have different requirements, combination on the same platform is 
feasible. The ACME and LDF experiments could be combined on a spacecraft on a GTO orbit with preferably an auxiliary diagnosis spacecraft.

The space environment offers the possibility to perform experiments on very extended and rarefied, weakly ionized plasmas, whose physics is still in large part to be investigated even from the point of view of numerical simulation. A strong element of originality of this kind of experiments with respect to already performed and even just proposed active plasma experiments is the focus on collisional and atomic physics. Specific experiments are proposed in this report which look promising not only in themselves but also in terms of mutual integration in a single satellite, including plasma generation, diagnostics, hydrodynamic wave generation and excited state kinetics.

\section{Acknowledgments}

This work has been supported by ESA under contract ESTEC AO/1-4615/04/NL/LvH. The authors wish to thank Professors K H Hassouni and K Lackner for discussions and many useful suggestions. One of the authors (JTM) thanks L Gargate for running the hybrid code.

\section{References}

[1] Bruno D, Capitelli M, Ceccherini F, Daly E, de Pascale O, Hilgers A, Longo S, Marcuccio S, Pegoraro F and Sanmartin J 2006 Proc. 36th COSPAR Scientific Assembly (Beijing) (ESA Contract ESTEC AO/1-4615/04/NL/LvH Final report)

[2] Rietveld M T 1998 Active experiments in space plasmas Proc. DO.6 Symp. of COSPAR Scientific Commission D (31st COSPAR Scientific Assembly, Birmingham, UK, 1996) ed M T Rietveld (New York: Pergamon)

[3] Oraevsky V N and Tríska P 1993 Active plasma experiment-Project APEX Adv. Space Res. 13 103-11

[4] Biriukov A S, Veselovskii I S, Grigorian O R, Kuznetsov S N and Nikitskii 1990 The magnesphere experiment using a source of intense magnetic field in the ionosphere Cosmol. Res. 28 955-8

[5] Biancalani A 2005 Thesis University of Pisa

[6] Biriukov A S, Veselovskii I S, Grigorian O R, Koval' A D, Kuznetsov S N, Kropotkin A P, Panasiuk M I, Riabukha S B, Us A A and Shuvalov V A 1992 Investigation of magnetospheric processes with the use of a source of strong magnetic field in the ionosphere Adv. Space Res. 12 135-41

[7] Alpert I L 1974 Waves and Artificial Bodies in the Near-Earth Plasma (Moscow: Izdatel Nauka)

[8] Block L P 1976 Interpretation of laboratory experiments of interest to space physics NASA STI/Recon Technical Report No 77, 25046

[9] Yur G, Chang T-F, Rahman H U, Birn J and Chao C K 1999 Magnetotail structures in a laboratory magnetosphere J. Geophys. Res. 104 14517-28

[10] Mauel M E, Warren H H and Hasegawa A 1992 An experiment to measure collisionless radial transport of energetic electrons confined by a dipole magnetic field IEEE Trans. Plasma Sci. 20 626-30

[11] Kesner J and Mauel M 1997 Plasma confinement in a levitated magnetic dipole Plasma Phys. Rep. 23 742-50

[12] Simakov A et al 2002 Stability of axisymmetric plasmas in closed line magnetic fields 19th IAEA Conf. of Plasma Physics and Controlled Nuclear Fusion (Lyon, France, 2002) paper IC/P-13

[13] Hasegawa 1987 The use of a dipole magnetic field generated by a levitated ring to confine a hot plasma Comm. Plasma Phys. Control Fusion 1147

[14] Teller E et al 1992 Space propulsion by fusion in a magnetic dipole Fusion Technol. 22

[15] Mendonca J T 2005 The artificial magnetosphere concept IAGA Symp. (Toulouse) IAGA2005-A-01497

[16] Mendonca J T, Brinca A L, Fonseca R, Loureiro J, Silva L O and Vieira I 2005 Physical problems of artificial magnetospheric propulsion J. Plasma Phys. 71495

[17] Stenzel R L and Urrutia J M 1989 Whistler wings from moving electrodes in a magnetized laboratory plasma Geophys. Res. Lett. 16 361-4

[18] Urrutia J M and Stenzel R L 1990 Modeling of induced currents from electrodynamic tethers in a laboratory plasma Geophys. Res. Lett. 17 1589-92

[19] Stenzel R L and Urrutia J M 1989 Geophys. Res. Lett. 16361

[20] Matsumoto Y and Hoshino M 2006 Turbulent mixing and transport of collisionless plasmas across a stratified velocity shear layer J. Geophys. Res. 111 A05213 
[21] Yamada M 2001 Review of the recent controlled experiments for study of local reconnection physics Earth Planets Space $\mathbf{5 3} 509$

[22] Boris P 1970 Relativistic plasma simulation-optimization of a hybrid code Proc. 4th Conf. on Numerical Simulation of Plasmas (Washington, DC, 1970)

[23] Winglee R M, Slough J, Ziemba T and Goodson A 2000 Mini-magnetospheric plasma propulsion: tapping the energy of the solar wind for spacecraft propulsion J. Geophys. Res. 10521067

[24] Toivanen P K, Janhunen P and Koskinen H E J 2004 Magnetospheric propulsion (eMPii) ESTEC/Contract No. 16361/02/NL/LvH ESA Study on Magnetospheric Propulsion for Scientific Exploration(eMPii), Final Report

[25] Loureiro J, Mendonca J T, Brinca A L, Fonseca R, Silva L O and Vieira I 2005 J. Atmos. Sol.-Terr. Phys. 671315

[26] Conrads M and Schmidt M 2000 Plasma Sources Sci. Technol. 9441

[27] Sanna G and Tomassetti G 2005 Molecular Beams Gas Dynamics (London: Imperial College Press)

[28] Capitelli M, Ferreira C M, Gordiets B F and Osipov A 2005 Plasma Kinetics in Atmospheric Gases (Springer Series on Atomic, Optical, and Plasma Physics vol 31) (Berlin: Springer)

[29] Menzel D 1969 Astrophys. J. Suppl. 18221

[30] Shaver P 1975 Pramana J. Phys. 51

[31] Kaplan S and Pikel'ner S 1979 Physics of the Interstellar Medium (Moscow: Nauka)

[32] Sholin G V and Gulyaev S A 1977 Sov. Astron. AJ 54301

[33] Beigman I L 1977 Sov. Phys._JETP 731729

[34] Bruno D, Laricchiuta A, Capitelli M and Catalfamo C 2007 Effect of electronic excited states on transport in magnetized hydrogen plasma Phys. Plasmas 14022303

[35] Svelto O 1998 Principles of Lasers (Berlin: Springer)

[36] Johnson M H and Ball J K 1992 Combined release and radiation effects satellite (CRRES): spacecraft and mission J. Spacecr. Rockets 29556

[37] Crow J E, Auer P L and Allen J E 1975 J. Plasma Phys. 1465

[38] Mora P 2003 Phys. Rev. Lett. 90185002

[39] Peano F, Peinetti F, Mulas R, Coppaand G and Silva L O 2006 Phys. Rev. Lett. 96175002

[40] Annaratone B M, Ku V P T and Allen J E 1995 J. Appl. Phys. 775455

[41] Annaratone B M, Bandyopadhyay P, Chaudhuri M and Morfill G E 2006 New J. Phys. 8306

[42] Martinez-Sanchez M and Sanmartin J R 1997 J. Geophys. Res. 10227257

[43] Sanmartin J R, Charro M, Pelaez J, Tinao I, Elaskar S, Hilgers A and Martinez-Sanchez M 2006 J. Geophys. Res. 111 A 11310

[44] Sanmartin J R and Estes R D 1999 Phys. Plasmas 6395 